\title{
RETRACTED ARTICLE: Investigation of a Fatigue Resistant Retrofit Method for Highmast Luminaire Support Structures. Part I: Numerical Study
}

\author{
Yeun Chul Park ${ }^{1}$. Sougata Roy ${ }^{2} \cdot$ Reilly W. Thompson ${ }^{3}$
}

Received: 2 August 2016 / Accepted: 8 November 2017 / Published online: 20 April 2018

(c) Korean Society of Steel Construction 2018

The corresponding author has retracted this article as it is apparent that the article was submitted without permission of the named co-authors, and hence permission for copyright transfer was not obtained. All named authors agree to this retraction. The online version of this article contains the full text of the retracted article as electronic supplementary material.

Electronic supplementary material The online version of this article (https://doi.org/10.1007/s13296-018-0001-6) contains the full text of the retracted article as electronic supplementary material.

Yeun Chul Park

ryan1886@gmail.com

Reilly W. Thompson

rthompson@arorapc.com

1 Institute of Construction and Environmental Engineering,

Seoul National University, 1 Gwanak-ro, 32-303,

Gwanak-gu, Seoul 08826, Republic of Korea

2 Bethlehem, PA, USA

3 Arora and Associates, P.C., Lawrenceville, USA 